\title{
Celastrol and thymoquinone alleviate aluminum chloride-induced neurotoxicity: behavioral psychomotor performance, neurotransmitter level and oxidative-inflammatory burden in brain of rats
}

\section{Faten Abbas}

Suez Canal University

\section{Mohamed Ahmed Eladl}

University of Sharjah, Sharjah 27272, United Arab Emirates

Mohamed El-Sherbiny

College of Medicine, AlMaarefa University, Riyadh

Nadia Abozied

Faculty of Medicine, Suez Canal University

Amaal Nabil

Faculty of Medicine, Suez Canal University

Shereen M. Mahmoud

Faculty of Veterinary Medicine, Suez Canal University

Sawsan Zaitone ( $\sim$ sawsan_zaytoon@pharm.suez.edu.eg)

Suez Canal University

Dalia Ibrahim

Suez Canal Unversity

\section{Research Article}

Keywords: AlCl3, psychomotor activity, celastrol, inflammation, neurotransmitters, oxidative stress, thymoquinone

Posted Date: December 29th, 2021

DOI: https://doi.org/10.21203/rs.3.rs-1197175/v1

License: (c) (1) This work is licensed under a Creative Commons Attribution 4.0 International License. Read Full License 


\section{Celastrol and thymoquinone alleviate aluminum chloride-induced neurotoxicity: behavioral psychomotor performance, neurotransmitter level and oxidative-inflammatory burden in brain of rats}

Faten Abbas', Mohamed Ahmed Eladl ${ }^{*}$, Mohamed El-Sherbiny ${ }^{3}$, Nadia Abozied ${ }^{4}$, Amaal Nabil $^{4}$, Shereen M. Mahmoud ${ }^{5}$, Sawsan A. Zaitone ${ }^{6,7}$ * Dalia Ibrahim $^{1}$

${ }^{1}$ Physiology Department, Faculty of Medicine, Suez Canal University, Ismailia, Egypt. faten_abbas@med.suez.edu.eg. Dalia_ibrahim@med.suez.edu.eg

${ }^{2}$ Department of Basic Medical Sciences, College of Medicine, University of Sharjah, Sharjah 27272, United Arab Emirates; meladl@ sharjah.ac.ae

${ }^{3}$ Department of Basic Medical Sciences, College of Medicine, AlMaarefa University, Riyadh 71666, Saudi Arabia; msharbini@mcst.edu.sa

${ }^{4}$ Pharmacology Department, Faculty of Medicine, Suez Canal University, Ismailia, Egypt. Nadia_abouzaid@med.suez.edu.eg; Amalnabil@med.suez.edu.eg

${ }^{5}$ Forensic medicine \& Toxicology, Faculty of Veterinary Medicine, Suez Canal University, Ismailia, Egypt. Shereenammar@vet.suez.edu.eg

${ }^{6}$ Department of Pharmacology \& Toxicology, Faculty of Pharmacy, Suez Canal University, Ismailia 41522, Egypt. Sawsan_zaytoon@pharm.suez.edu.eg

${ }^{7}$ Department of Pharmacology \& Toxicology, Faculty of Pharmacy, University of Tabuk, Tabuk, Saudi Arabia.

* Corresponding author: MAE (meladl@sharjah.ac.ae) \& $\quad$ SAZ (Sawsan_zaytoon@pharm.suez.edu.eg; szaitone@ut.edu.sa 


\begin{abstract}
:
The exposure to metal aluminum such as aluminum chloride ( $\mathrm{AlCl} 3)$ induces inflammatoryoxidative reactions with progressive neurodegeneration in different brain regions in animal models. The current study was designed to assess the role of celastrol or thymoquinone (TQ) in alleviating $\mathrm{AlCl} 3$ induced behavioral psychomotor changes and oxidative-inflammatory burden in albino male rats. Four groups were used in this study, (i) vehicle control group, (ii) AlCL3 control group: rats received intraperitoneal injection (i.p.) of $\mathrm{AlCl} 3(10 \mathrm{mg} / \mathrm{kg})$, (iii) $\mathrm{AlCl} 3+\mathrm{TQ}$ (10 mg/kg, i.p.) group and (iv) $\mathrm{AlCl} 3+$ celastrol ( $1 \mathrm{mg} / \mathrm{kg}$, i.p.) group. In general, all injections remained for 6 weeks. Behavioral psychomotor evaluation (open field test, rotarod test and forced Swimming test) were done to assess locomotor, motor coordination, anxiety-like behavior and depressive-like behavioral. Markers of oxidative stress, malondialdehyde (MDA), total antioxidant capacity (TAC) and catalase enzyme activity (CAT) and the proinflammatory mediators, tumor necrosis factor- $\alpha(\mathrm{TNF}-\alpha)$ and interlukin-6 (IL-6) were measured in the rat brains. Neurotransmitters including acetylcholine (ACh), dopamine and serotonin in addition to acetylcholinesterase enzyme (AChE) level were measured in brain homogenates. Our results demonstrated that daily injection of TQ or celastrol significantly improved behavior psychomotor deficits, decreased AChE activity towards their normal levels. Tissue oxidative stress and proinflammatory markers were modulated by TQ and celastrol. These results concluded that TQ and celastrol have useful in alleviating $\mathrm{AlCl} 3$-induced neurotoxicity by their antioxidant and anti-inflammatory properties. Hence, they are looking promising for investigating their preventive effect in animal models of neurodegenerative diseases.
\end{abstract}

Keywords: $\mathrm{AlCl} 3$; psychomotor activity; celastrol, inflammation; neurotransmitters; oxidative stress; thymoquinone. 


\section{Introduction}

Neurotoxicity usually occurs when the nervous system is exposed to a variety of toxic compounds, such as aluminum, and manifests as psychomotor and cognitive symptoms (Troshin 2009). Aluminum has no biological function in the human body and is known to cause neurotoxicity, which has been linked to the onset of dialysis encephalopathy. Some dialysis patients are exposed to Aluminum in the dialysis fluid, resulting in neurobehavioral symptoms, seizures, and death (Baydar et al. 2003). In animal models, exposure to metal aluminum, such as aluminum chloride, caused inflammatory reactions and neuronal abnormalities at the synapse level, as well as progressive neurodegeneration in various brain and spinal cord regions. Aluminum has the ability to cross the blood-brain barrier, causing central nervous system functioning and behavioral problems (Baydar et al. 2003, Bhalla et al. 2010, Yokel 2000). There are numerous evidences that suggest that aluminum exposure and accumulation in the brain is linked to chronic debilitating, incurable, and highly prevalent neurodegenerative disorders such as Parkinson's (PD) and Alzheimer's (AD) by altering neurotransmitter metabolism, such as cholinergic system function, which is highly susceptible to aluminum, accumulating iron, promoting iron-dependent lipid peroxidation, and producing reactive oxygen species. However, these conclusions are still debatable and unclear (Ghorbel et al. 2016, Gitler et al. 2017, Skalny et al. 2021, Yokel 2000). Aluminum exposure may cause alterations in the levels of several neurotransmitters that are tightly linked. In addition, Aluminum influences the metabolism of various neurotransmitters in the brain of animals, including acetylcholine (ACh), serotonin, and dopamine, which has an impact on behavioral responses (Bhalla et al. 2010, Kumar \&toxicology 2002, Skalny et al. 2021). Many neurotransmitter levels that are intimately linked may vary as a result of aluminum exposure (Kumar \&toxicology 2002). It is critical to investigate the mechanisms of its neurotoxicity, which remain controversial in animal models, and to suspect a useful therapeutic approach to aluminum, a highly toxic metal with widespread distribution in human environments (water, food, drugs, cookware, and industrial sources), as well as remaining living organisms, for the prevention and treatment of its neurotoxicity (Brough \&Jouhara 2020, Skalny et al. 2021).

Recently, the use of antioxidant agents derived from plant extracts and diet has been shown to be a promising therapeutic approach for preventing metal toxicity and neurodegenerative disorders linked to oxidative stress and inflammation, which activate microglia, causing increased production of proinflammatory cytokines, reactive oxygen species, and lipid peroxidation, and eventually apoptosis (Al-Otaibi et al. 2018, Allison et al. 2000).

Thymoquinone (TQ), a redox-active quinone oil obtained from Nigella sativa seeds (black cumin), possesses polyphenol characteristics and is generated from Nigella sativa seeds (black cumin). Anti-inflammatory and antioxidant properties, as well as the ability to pass the blood-brain barrier, scavenge free radicals and prevent cell damage caused by oxidative chemicals (Ahmad et al. 2013, Elmaci et al. 2016, Hosseinzadeh et al. 2012). In addition, it exhibits anti-anxiety and antidepression properties in rats (Elmaci et al. 2016). Furthermore, due to its significant antioxidant and anti-inflammatory qualities, it has a neuroprotective impact in numerous neurological disorders such as PD and epilepsy (Majdalawieh et al. 2010). Its neuroprotective impact against heavy metals and radiation exposure has been demonstrated in a number of investigations (Elmaci et al. 2016, Kassab et al. 2017, Majdalawieh et al. 2010). 
Celastrol is an antioxidant herbal plant that chelates metals like calcium and cadmium while quenching reactive oxygen species (Zhang et al. 2017). It is a quinone and phenolic methide triterpenoid isolated from the Tripterygium wilfordii Hook F (TWHF) plant, and it's one of the most popular natural medicine compounds, especially in China, for its anti-inflammatory and antioxidant properties (Li \&Hao 2019). Celastrol has been proposed as a potential preventative and treatment for neurodegenerative disorders due to its antioxidant and anti-inflammatory effects (Li \&Hao 2019, Paris et al. 2010). Celastrol also protected animal models from locomotor dysfunction and social behavior impairment (Bove et al. 2020).

The current study was designed to assess the effects of TQ and Celastrol, two effective antioxidant and anti-inflammatory herbal plants, in aluminum chloride $(\mathrm{AlCl} 3)$ induced neurotoxicity in rats, with a focus on behavioral psychomotor performance, neurotransmitters changes, and oxidativeinflammatory burden in whole brain of albino rats.

\section{Materials and Methods}

\section{Animals}

Twenty-four adult male albino Wister rats weighing 175-187 g were used in this study. Animals were purchased from The Ophthalmology Research Institute (Giza, Egypt); they were housed in spacious wire mesh cages at room temperature and were kept with free access to standard rat chow diet and tap water. Rats were left for acclimatization for one week before the start of the study. Any animals with overt signs of illness were removed from the study. All experiments approved by the committee for animal care and ethics at the medical college of Suez Canal University with approval No. 4741.

\section{Chemicals}

AlCl3, TQ and celastrol were purchased from Sigma-Aldrich Co. Weekly stock fresh solutions were prepared as follow: ALCL3 was dissolved in distilled water however, TQ and celastrol were dissolved in $1 \%$ dimethyl sulfoxide (DMSO).

\section{Groups and design of the study}

The rats were randomly divided into 4 groups of 6 rats. Group I (normal group): rats were given $1.0 \mathrm{ml}$ normal saline once daily, Group II (AlCl3 group): group of neurotoxicity, AlCl3 (10 $\mathrm{mg} / \mathrm{kg} /$ day) was given intra peritoneal (i.p.) once daily and this dosing regimen of $\mathrm{AlCl} 3$ was selected based on a previous report because of its high rate of induction and low mortality (Khan et al. 2013), Group III (AlCl3+TQ group): TQ was concomitantly administered (10 mg/kg/day, i.p.) once daily with $\mathrm{AlCl} 3$ (10 mg/kg/day) (Al-Ali et al. 2008, Ince et al. 2013) and Group IV (AlCl3+Celastrol group): Celastrol was concomitantly administered by i.p. injection (1 $\mathrm{mg} / \mathrm{kg} /$ day); this is the dose recommended in models of neurodegenerative diseases once daily (Paris et al. 2010).

There was a week of acclimatization followed by a period of 6-weeks for the rat experiment. The drug preparation and injection were carried out under aseptic conditions and recorded the body

weight changes by electronic animal weight scale of all the rats at the beginning of the experiment 
as the initial record for rats, then finally before sacrificing them to calculate the final weight for each rat.

\section{Behavioral evaluation}

\section{The open-field test for evaluation of locomotor behavior}

The open-field arena $(100 \times 100 \times 40 \mathrm{~cm})$ was made of wood. The floor was painted with white lines that formed 25 squares $(20 \times 20 \mathrm{~cm})$ pattern. Rats were introduced individually in the open field arena and rat behavioral parameters were observed and video-recorded for 5-min sessions; the test was performed under high-light conditions. Rats were observed for ambulation (the number of squares crossed; only when the animal enters a square with both its forelimb (one count is made). An experienced observer, who was blinded to the treatment groups, quantified the open-field locomotor behavior. In addition, number of rearing; i.e. standing on hind limbs (vertical movement) and leaning on the wall with forelegs, and looking around were also observed (Zghari et al. 2018).

\section{The rotarod test for evaluation of motor coordination}

Animals were monitored for motor coordination and balance with the help of rotarod apparatus (3 $\mathrm{cm}$ in diameter and rotating at a constant speed of 20rpm). Initially, animals were habituated to maintain posture on the rotarod by giving two training sessions of 5 min each with a gap of 10 min between the two sessions. After training, animals were allowed to move over the rotarod and their falling number was recorded per $5 \mathrm{~min}$ (Metz et al. 2005).

\section{The open-field test for anxiety-like behavior evaluation}

Another arena of 25 squares was divided into 9 central and 16 peripheral squares. At the beginning of the 5 min session, the rat was put in the center of the arena and its behavior was registered by mobile camera for manual analysis. The parameters of time spent by rats in the center of the arena (anxiolytics area) and in the peripheral area (anxiogenic area) were calculated and were used as a measure of anxiety level (Zghari et al. 2018).

\section{Forced swimming test for depressive-like behavioral}

The session of the forced swimming test was started by putting the rat in a cylindrical container $\left(50 \times 20 \mathrm{~cm}\right.$ ) filled with $30 \mathrm{~cm}$ of $22^{\circ} \mathrm{C}$ water for $5 \mathrm{~min}$ ). The time spent immobile (when rats stopped all active behaviors and became inactive floating) was considered as an index of depression-like behavior. High time of floating was analyzed as an increased depressive-like response (Rashwan et al. 2018).

\section{Preparation of brain homogenates}

The whole brains were dissected out from the rats after sacrificed them at the end of study duration and psychomotor evaluation by decapitation, cleared of the adhering tissues, weighed, washed with cold phosphate buffer saline and homogenized in normal saline $(10 \% \mathrm{~W} / \mathrm{V})$. The homogenates were centrifuged at $3000 \mathrm{rpm}$ for $10 \mathrm{~min}$. The resulting supernatant was kept at- $80{ }^{\circ} \mathrm{C}$ for the following measurements (Al-Otaibi et al. 2018). 


\section{Neurotransmitters evaluation}

$\mathrm{ACh}$ and its hydrolysis enzyme, AChE in the brain supernatant homogenate were measured by the using a choline/acetylcholine assay kit (Bio Vision Inc., Co., California, USA, catalog \#: K615) as a quantification colorimetric method. Also, brain AChE level was evaluated calorimetrically by a kit from BioVision, (catalog \#: k764). In addition, serotonin and dopamine were measured colorimetrically and their ELISA kits also from (BioVision Inc., co, California, USA, catalog \#: E4294 and K4219) respectively.

\section{Measurement of brain oxidant-antioxidant markers}

Brain lipid peroxidation was measured as MDA (nmol/g tissue) in the supernatant of the homogenized brain by the thiobarbituric acid colorimetric method using the MDA assay kit to make a pink colored complex with absorbance level at $532 \mathrm{~nm}$ from Cell Biolabs (USA, Catalog \#: STA-832). On the other hand, the TAC was evaluated by a colorimetric method based on the ability of brain antioxidants to suppress the production of thiobarbituric acid reactive substances (TBARS) by using ELISA kit (Cell Biolabs, USA, Catalog No.: sta-360). CAT was measured in the supernatant of the homogenized brain by ELISA kit from Cusabio Co. (Wuhan, China, Catalog No.: CSB- E13439r).

\section{Measurement of brain inflammatory markers}

TNF- $\alpha$ and IL-6 levels: these levels were measured in the brain homogenate using a rat TNF- $\alpha$ ELISA kit from Cusabio Co. (Wuhan, China, Catalog No.: CSB-E11987r) and a rat IL-6 ELISA kit from the same company, (Catalog No.: CSB- E04640r) use the quantities sandwich based immunoassay technique to measure them. We followed the steps and instructions of all previous kits that were used in the present study according to their manufacturers.

\section{Statistical analysis}

The data were presented as means \pm standard deviation (SD). Statistical Analysis of data was done using the SPSS package (Inc., Chicago, USA) and the variance of one way (ANOVA) for nonparametric data to elucidate differences between all groups. Post hoc range test (Bonferroni's) was used to test the difference between each pair of means. The level of significance was at $\mathrm{p}<0.05$.

\section{Results}

\section{Animal body and brain weights}

The final animal's body weight showed a significant decrease in the $\mathrm{AlCl} 3$ group than the control group. $\mathrm{AlCl} 3+\mathrm{TQ}$ and $\mathrm{AlCl}+$ +celastrol groups showed a significant gain in final body weight than the $\mathrm{AlCl} 3$ group with significant superiority for TQ. The initial body weight and brain weight showed non-significant change in all groups (Table 1). 
Table 1. Effect of AICl3, TQ and Celastrol on body and brain weights.

\begin{tabular}{lccc}
\hline Groups & $\begin{array}{c}\text { Initial body weight } \\
(\mathbf{g})\end{array}$ & $\begin{array}{c}\text { Final body weight } \\
(\mathbf{g})\end{array}$ & $\begin{array}{c}\text { Brain weight } \\
(\mathbf{g})\end{array}$ \\
\hline Control & $180.22 \pm 3.79$ & $270.4 \pm 4.19$ & $2.11 \pm 0.41$ \\
\hline AlCl3 & $178.99 \pm 3.34$ & $239.2 \pm 3.54^{\mathrm{a}}$ & $1.8 \pm 0.24$ \\
\hline AlCl3+TQ & $177.23 \pm 1.27$ & $262.3 \pm 5.44^{\mathrm{ab}}$ & $1.9 \pm 0.19$ \\
\hline AlCl3+Celastrol & $179.42 \pm 3.01$ & $250.7 \pm 4.92^{\mathrm{abc}}$ & $1.92 \pm 0.07$
\end{tabular}

Results were expressed as mean \pm SD and analyzed using one-way ANOVA followed by Bonferroni's post-hoc test at $\mathrm{P}<0.05 . \mathrm{a}, \mathrm{b}$ and $\mathrm{c}$ Represents a statistically significant difference when compared to Control, $\mathrm{AlCl} 3$ and $\mathrm{AlCl} 3+\mathrm{TQ}$, respectively.

\section{Behavior and locomotor tests}

\section{Evaluation of locomotor function by the open field test}

Behavioral locomotor performances of rats were evaluated by in the open field test as horizontal and vertical ambulation. There was a significant decrease in horizontal and vertical ambulation in the $\mathrm{AlCl} 3$ group as compared to the control group $(34 \pm 7.29$ vs. $90 \pm 9.48)$. $\mathrm{AlCl} 3+\mathrm{TQ}$ and $\mathrm{AlCl} 3+\mathrm{Celastrol}$ groups significantly increased the results as compared to the $\mathrm{AlCl} 3$ group $(78 \pm 8.81$ and $69 \pm 8.24$, respectively). There was no significant difference in horizontal and vertical ambulation between $\mathrm{AlCl} 3+\mathrm{TQ}$ and $\mathrm{AlCl} 3+$ celastrol groups (Fig 1).

\section{Rotarod test}

The motor coordination and balance level of rats were evaluated by a rotarod test. There was a significant difference between all groups regarding the number of falling down in the rotarod test. AICI3 produced disturbance in coordination of movement (number of falls $=14.53 \pm .3 .21$ vs. $4.32 \pm 2.08$ in the control group). $\mathrm{AlCl} 3+\mathrm{TQ}$ and $\mathrm{AlCl} 3+$ celastrol groups showed better performance and lower number of falls with significant superiority for celastrol $(9.56 \pm 1.86$ and $6.01 \pm 1.47$, respectively, Fig 2).

\section{Anxiety-like behavior in the open field test}

Anxiety level was evaluated in the open field as appeared in $\mathrm{AlCl} 3$ group that showed significant higher level of behavioral anxiety pattern than the control group [decreased spent time in central zone (anxiolytic area) and decreased grooming activity] (88 $.22 \pm 7.41$ vs. $145.31 \pm 13.35 \&$ $2.21 \pm 1.47$ vs. $11.33 \pm 3.41$, respectively). But all parameters of anxiety were significantly improved in $\mathrm{AlCl} 3+\mathrm{TQ}$ and $\mathrm{AlCl} 3+\mathrm{Celastrol}$ groups with significant superiority for TQ (Fig 3).

\section{Depressive-like behaviors in the rat swimming test}

The depression level of rats was measured by calculating the time of stability (immobility time) during physical activity in force swimming test. There was significantly increased immobility time in the $\mathrm{AlCl} 3$ group as compared to the normal group (16.23 \pm 2.19 vs. $3.41 \pm 1.37)$. $\mathrm{AlCl} 3+\mathrm{TQ}$ and $\mathrm{AlCl} 3+$ Celastrol significantly decreased this time $(8.01 \pm 1.79$ and $11.91 \pm 2.76$, respectively) as compared to $\mathrm{AlCl} 3$ group with no significant difference between $\mathrm{AlCl} 3+\mathrm{TQ}$ and $\mathrm{AlCl} 3+\mathrm{Celastrol}$ groups (Fig 4). 


\section{AChE enzyme and neurotransmitter evaluation}

There was a significant increase in acetylcholine esterase ( $\mathrm{AChE}$ ) level in rat brains in $\mathrm{AlCl} 3$ group when compared with normal group. TQ and celastrol improved its level when compared with $\mathrm{AlCl} 3$ group with no significant difference between them. There was a significant decrease in ACh level in rat brains in $\mathrm{AlCl} 3$ group when compared with normal group. $\mathrm{AlCl} 3+\mathrm{TQ}$ and $\mathrm{AlCl} 3+$ celastrol improved its level when compared with $\mathrm{AlCl} 3$ group with no significant difference between them (Table 2).

Table 2. AChE and ACh level in the homogenate brain tissue.

\begin{tabular}{|c|c|c|}
\hline Groups & $\begin{array}{l}\mathrm{AChE} \\
(\mathrm{nmol} / \mathrm{min} / \mathrm{mg} \text { of tissue) }\end{array}$ & $\begin{array}{l}\text { ACh } \\
\text { (nmol/mg of brain tissue) }\end{array}$ \\
\hline Control & $12.5 \pm 1.95$ & $48.34 \pm 2.79$ \\
\hline AlCl3 & $30.71 \pm 2.87^{\mathrm{a}}$ & $21.21 \pm 1.76^{\mathrm{a}}$ \\
\hline AlCl3+TQ & $17.45 \pm 2.89^{\mathrm{ab}}$ & $39.32 \pm 1.73^{\mathrm{ab}}$ \\
\hline AlCl3+Celastrol & $15.82 \pm 1.11^{\mathrm{ab}}$ & $37.41 \pm 2.01^{\mathrm{ab}}$ \\
\hline
\end{tabular}

There was significantly decreased in dopamine level in rat brains in $\mathrm{AlCl} 3$ group when compared with normal group. $\mathrm{AlCl} 3+\mathrm{TQ}$ and $\mathrm{AlCl} 3+\mathrm{Celastrol}$ improved its level towards normal level when compared with $\mathrm{AlCl} 3$ group with no significant difference between them. In addition, there was significantly decreased in serotonin level in brain rats of $\mathrm{AlCl} 3$ group when compared with control group. $\mathrm{AlCl} 3+\mathrm{TQ}$ and $\mathrm{AlCl} 3+$ Celastrol improved the dopamine and serotonin levels when compared with $\mathrm{AlCl} 3$ group with significant superiority for TQ (Table 3).

Table 3. Dopamine and serotonin levels in the homogenate brain tissue.

\begin{tabular}{|c|c|c|}
\hline Groups & $\begin{array}{c}\text { Dopamine level } \\
\text { (ng/g of brain tissue) }\end{array}$ & $\begin{array}{c}\text { Serotonin level } \\
\text { (ng/g of brain tissue) }\end{array}$ \\
\hline Control & $489.931 \pm 4.47$ & $413.228 \pm 1.81$ \\
\hline AlCl3 & $267.511 \pm 7.62^{\mathrm{a}}$ & $215.772 \pm 7.73^{\mathrm{a}}$ \\
\hline AlCl3+TQ & $375.421 \pm 14.04^{\mathrm{ab}}$ & $352.881 \pm 7.38^{\mathrm{ab}}$ \\
\hline AlCl3+Celastrol & $363.322 \pm 14.56^{\mathrm{ab}}$ & $298.845 \pm 11.74 \mathrm{abc}$ \\
\hline
\end{tabular}




\section{Oxidative-antioxidative markers}

Lipid peroxidation (LPO) as an oxidative marker in brain tissue was measured by its malondialdehyde (MDA) content. The level of MDA significantly increased in the $\mathrm{AlCl} 3$ group when compared to the control group. $\mathrm{AlCl} 3+\mathrm{TQ}$ and $\mathrm{AlCl} 3+\mathrm{Celastrol}$ groups showed lower MDA level which is significantly different compared with $\mathrm{AlCl} 3$ group with the superiority for TQ. The level of catalase (CAT) was significantly decreased in the $\mathrm{AlCl} 3$ group when compared to the control group. TQ and celastrol improved the CAT level with significant differences with the $\mathrm{AlCl} 3$ group and the superiority for celastrol. The total antioxidant capacity (TAC) was significantly decreased in the $\mathrm{AlCl} 3$ group when compared to the control group. TQ and Celastrol improved its level with significant differences with the $\mathrm{AlCl} 3$ group and the superiority for celastrol (Table 4).

Table 4. MDA, CAT and TAC levels in the brain tissue homogenates.

\begin{tabular}{|c|c|c|c|}
\hline Groups & $\begin{array}{c}\text { MDA } \\
\text { (nmol/g of brain } \\
\text { tissue })\end{array}$ & $\begin{array}{c}\text { CAT } \\
(\mu \mathrm{M} \text { of } \\
\mathrm{H}_{2} \mathrm{O}_{2} \text { decomposed } / \mathrm{min} / \mathrm{mg} \\
\text { of brain tissue })\end{array}$ & $\begin{array}{c}\text { TAC } \\
(\mu \mathrm{mol} / \mathrm{g})\end{array}$ \\
\hline Control & $160.56 \pm 3.73$ & $45.34 \pm 3.67$ & $775.31 \pm 20.28$ \\
\hline AlCI3 & $270 \pm 8.36^{\mathrm{a}}$ & $9.86 \pm 2 . .56^{\mathrm{a}}$ & $219.42 \pm 4.86^{\mathrm{a}}$ \\
\hline AlCl3+TQ & $230.32 \pm 8.78^{\mathrm{ab}}$ & $26.38 \pm 2.46 .^{a b}$ & $533.56 \pm 17.86^{b}$ \\
\hline AlCl3+Celastrol & $200.74 \pm 8.82^{\mathrm{abc}}$ & $39 \pm 1.82^{a b c}$ & $570.26 \pm 31.05^{a b c}$ \\
\hline
\end{tabular}

Results were expressed as mean \pm SD and analyzed using one-way ANOVA followed by Bonferroni's post-hoc test at $\mathrm{P}<0.05 . \mathrm{a}, \mathrm{b}$ and $\mathrm{c}$ Represents a statistically significant difference when compared to control, $\mathrm{AlCl} 3$ and $\mathrm{AlCl} 3+\mathrm{TQ}$ respectively.

Further, there was a significant increase in interlukin-6 (IL-6) and tumor necrosis factor- $\alpha$ (TNF$\alpha$ ) in the $\mathrm{AlCl} 3$ group as compared to the control group. TQ and Celastrol significantly decreased the levels of these inflammatory markers as compared to the $\mathrm{AlCl} 3$ group. $\mathrm{AlCl} 3+\mathrm{Celastrol}$ group showed significant improvement in these inflammatory markers compared to the $\mathrm{AlCl} 3+\mathrm{TQ}$ group (Table 5).

Table 9. Inflammatory markers in the brain homogenates.

\begin{tabular}{|c|c|c|}
\hline Groups & IL-6 (pg/mg) & TNF- $\alpha(\mathrm{pg} / \mathrm{mg})$ \\
\hline Control & $30.430 \pm 3.94$ & $20.102 \pm 5.26$ \\
\hline AlCl3 & $130.341 \pm 5.58^{a}$ & $100.163 \pm 7.51^{a}$ \\
\hline AlCl3+TQ & $70.025 \pm 6.87^{\mathrm{ab}}$ & $55.902 \pm 5.58^{\mathrm{ab}}$ \\
\hline AlCl3+Celastrol & $57.301 \pm 2.88^{a b c}$ & $40.040 \pm 3.54^{a b c}$ \\
\hline
\end{tabular}




\section{Discussion}

Exposure to $\mathrm{AlCl} 3$ could alter blood brain permeability and induce neuronal structure changes and inflammatory-oxidative responses (Baydar et al. 2003). Molecules extracted from plants are now considered as complementary and /or alternative therapies against various neurodegenerative disorders that are associated with inflammatory- oxidative stress (Dai et al. 2019). The neurotoxicity effects of $\mathrm{AlCl} 3$ and neuroprotective effects of TQ and Celastrol are still not yet completely known. The current study was designed to investigate the psychomotor, some neurotransmitters and inflammatory-oxidative responses induced by alc13 and the neuropreventive effects of TQ and Celastrol in albino rats.

Our findings showed that chronic exposure to $\mathrm{AlCl} 3$ produced a significant loss of final body weight when compared to the normal group. This result is in line with reports of other researchers who explained this loss on the basis of general toxicity of aluminum that affects energy metabolism through inhibition of glycolysis and Krebs cycle with promotion of lipid and protein oxidation (Han et al. 2013). The co- administration of TQ and Celastrol with AlCl3 significantly attenuated the loss in the final body weight that occurred by $\mathrm{AlCl} 3$ alone.

In the current study, the psychomotor behavioral responses in the $\mathrm{AlCl} 3$ group were assessed. In the open field test, the data from $\mathrm{AlCl} 3$ group revealed that the aluminum - toxicity significantly decreased the ambulation functions which are the number of squares crossed and the rearing movements compared to the control group. Similar observations were reported by Golub and Germann who noticed that the behavioral loco-motor activities significantly reduced after Aluminum administration by different doses (small, moderate and large) of aluminum (Golub et al. 2001). Moreover, there was a significant difference in the $\mathrm{AlCl} 3$ group regarding the number of falls in the Rotarod test when compared to the control group. Justin-Thenmozhi et al. reported significant decreases in the retention time of rotarod test with $\mathrm{AlCl} 3$ intoxication due to inflammatory markers such as (1L-6 and TNF- $\alpha$ ) and oxidative stress (Justin-Thenmozhi et al. 2018).

The significant anxiogenic action of the $\mathrm{AlCl} 3$ toxicity was reported in the current study by parameters of the anxiety-like behavior of open field test when compared with the normal group and this finding is corroborated by several studies that investigated the aluminum effects on behavior in animals. A study on adult mice showed that the aluminum had anxiogenic action by decreasing the time spent in the central area of the open field (Bannon 2015). Depression was also shown in the $\mathrm{AlCl} 3$ group of our study as significantly increasing the stability time parameter in forced swimming test that reflects depressive- like behavior effect of aluminum toxicity. In a Wistar adult male rat model, the $\mathrm{AlCl} 3$ significantly increased immobility time parameter confirming its depressive like-behavioral action (Rashwan et al. 2018). The mechanism by which aluminum induces this behavioral change in animals is not yet known. Depression might link to hypo-function of the central serotonergic system and serotonin level reduction in brain regions (Ravi et al. 2000).

Regarding the inflammatory markers, in the present study, the $\mathrm{AlCl} 3$ group significantly increased the level of TNF- $\alpha$ and IL-6, the potent inflammatory cytokines in the homogenate brain of rats when compared with the control group. Milnerowicz et al. reported that the metal exposure such 
as aluminum increased inflammatory cytokine TNF- $\alpha$ and some types of IL- 6 and IL- $1 \beta$ which stimulate leukocytes recruitment and as the end result, this recruitment potentiates the inflammation stress by more releasing of pro-inflammatory cytokines (Milnerowicz et al. 2015). In addition, when the ACh is down regulated to a lower level in brain, the inflammatory system is activated by enhancing the expression of N-methyl-D-aspartate receptor, hence the secretion of inflammatory cytokines are increased that having anticholinergic activity forming vicious cycle augmenting the neurotoxicity of aluminum and more brain damage (Hori et al. 2013). This relation between inflammatory cytokines and lower levels of ACh with higher levels of its catalytic enzyme $\mathrm{AChE}$ is in line with our results. Aluminum neurotoxicity effects in previous studies produced imbalance between generation of ROS and antioxidants and induced oxidative stress in neurons. Regarding oxidative stress, we measured LPO as MDA level and quantified the endogenous antioxidant CAT enzyme in the homogenate brain of rats. Now, it is well documented that the brain has great risk to be injured by oxidative stress and LPO due to its high content of lipids (Belaiid-Nouira et al. 2012). Our result showed a significant increase in MDA level in the homogenate brain of rats of the $\mathrm{AlCl} 3$ group when compared with the normal group and this finding reflects the potential role of aluminum in potentiation of lipid peroxidation, oxidative stress and brain damage on the basis of previous data.

In the present study, the AChE enzyme was significantly increased whereas the level of ACh decreased in brain rats of the $\mathrm{AlCl} 3$ group when compared to the normal group. These findings are in agreement with several studies, but also are in disagreement with another. These discrepancies between studies are explained by Peng et al. (Peng et al. 1992) who related them to the metal exposure duration, type of its salt and the difference in response of brain regions to aluminum toxicity. Aluminum might have caused lesions in the important regions of the brain like substantia nigra, cerebrum and hippocampus whereas little change was observed in other brain areas.

Dopamine neurotransmitter level was also measured and the rats of the $\mathrm{AlCl} 3$ group had significantly lower levels of dopamine in the brain when compared to the control group. Oxidative stress and hydrogen peroxide $(\mathrm{H} 2 \mathrm{O} 2)$ attenuates dopamine release and increases the loss of dopaminergic cells (Spanos et al. 2013). These previous observations formulate an attractive strategy to prevent or delay the progression of neurodegenerative diseases by controlling oxidative stress and the inflammatory response and this strategy is supported by Kato et al. (A Kato et al. 2013). Bowdler et al. (1979) (Bowdler et al. 1979) mentioned the fact that the serotonin has the important function in coordination between autonomic and neuroendocrine systems to control sensor- motor output, and this function explained the impairment of visuo-motor coordination performance by neural toxic inhibitory effect of aluminum on serotonin system. This fact may be partly explained the changes in psychomotor behavior tests in the present study such as the impairment in motor coordination in rotarod test and anxiety behavior in open field test in $\mathrm{AlCl} 3$ group. There is direct relationship between serotonin and dopamine and when the level of serotonin increased led to an increased in level of dopamine and vice versa (Yadid et al. 1994).

All neurotoxicity effects of $\mathrm{AlCl} 3$ were improved by TQ and celastrol in this study. TQ is present in Nigella sativa and has phenolic major bioactive components and potent antioxidant, Scavenge free radicals and anti-inflammatory activities against drug and chemicals induced multiple organ 
toxicities (Ahmad et al. 2013). TQ improved the psychomotor performance, lipid peroxidation and CAT level in this study. TQ improved the muscle coordination and spontaneous locomotor activity, decreased AChE activity and the level of lipid peroxidation, and increased levels of antioxidant enzymes i.e., reduced glutathione, CAT, in a rat model pretreated with chlorpromazine toxicity (Safhi 2016). In accordance with our results some findings showed a significant decrease in IL-6, and TNF- $\alpha$ in brain rats of TQ group and attenuates depression-like behavior. These findings are also in agreement with previously obtained results (Kassab et al. 2017) who observed that TQ decreased the levels of MDA, TNF- $\alpha$ and increased the levels of CAT, in the cerebral cortex, cerebellum, and brain stem.

In the current study, TQ exerted significantly anticholinesterase activity and improved ACh level, and this finding is in line with Abulfadl et al. (2018) (Abulfadl et al. 2018). TQ significantly increased TAC and decreased MD, TNF- $\alpha$ level. These results indicate that TQ holds as a neuroprotector for the treatment of neurodegenerative disorders (Abulfadl et al. 2018). On the previous data, TQ may be a promising approach for the prevention and therapy of neurodegenerative disorders due to $\mathrm{AlCl} 3$ toxicity by modulating the levels of neurotransmitters and reducing inflammatory- oxidative stress and improving the psychomotor behavior that all impaired by $\mathrm{AlCl} 3$ toxicity in the present study.

Celastrol is a traditional Chinese herbal with potent anti-inflammatory and anti-oxidative effects and has beneficial effects in the treatment of neurodegenerative, and inflammatory diseases (Bian et al. 2016). Celastrol treatment significantly overcome the depletion in dopamine, attenuated the concentration of neuro-inflammation and improved the motor deficits. Celastrol also corrected the impairments in horizontal movements and vertical activity in electronic maze that were analyzed by Osaka University Computerized system and improved psychomotor coordination was evaluated in a rotarod apparatus of Female Sprague-Dawley rat model (Allison et al. 2000). Celastrol corrected the loco- motor behavior and decreased immobility time in the forced swimming test and tail suspension test in mouse model and increased allo-grooming with inhibition of proinflammatory (TNF- $\alpha$, IL-6) in mice model (Bove et al. 2020, Zhu et al. 2021). Furthermore, Celastrol was found to be effective in another animal model of depression, such as the winter depression-like in fish by counterbalancing the increase in cortical ROS production, lipid peroxidation and inflammation (Nakayama et al. 2020). These previous benefits of Celastrol because of its phenol and carboxyl groups that scavenge free radicals and inhibit the peroxidation of the outer and inner mitochondrial membranes (Chen et al. 2014).

\section{Conclusions}

It is concluded that $\mathrm{AlCl} 3$ may be attributed to oxidative-inflammatory stress, disturbances of psycho - motor behaviors and some neurotransmitter levels. The results suggested that the TQ and celastrol were able to antagonize $\mathrm{AlCl} 3$ neurotoxicity at least partly, by their potent antioxidant and anti-inflammatory properties as well as by neurotransmitter modulation. Hence, TQ and celastrol are promising agents for investigating their preventive effect in animal models of neurodegenerative diseases. 


\section{Author Contributions:}

Conceptualization, Faten Abbas and Dalia Ibrahim; Data curation, Nadia Abozied, Amal Nabil and Shereen Mahmoud; Formal analysis, Faten Abbas and Dalia Ibrahim; Investigation, Faten Abbas and Dalia Ibrahim; Methodology, Faten Abbas and Dalia Ibrahim; Resources, Faten Abbas, Mohamed Ahmed Eladl, Mohamed El-Sherbiny, Nadia Abozied, Amal Nabil, Shereen Mahmoud, Sawsan Zaitone and Dalia Ibrahim; Software, Mohamed Ahmed Eladl and Sawsan Zaitone; Supervision, Faten Abbas; Validation, Mohamed El-Sherbiny; Visualization, Mohamed Ahmed Eladl, Mohamed El-Sherbiny, Nadia Abozied, Amal Nabil, Shereen Mahmoud, Sawsan Zaitone and Dalia Ibrahim; Writing - original draft, Faten Abbas, Nadia Abozied, Amal Nabil, Shereen Mahmoud and Dalia Ibrahim; Writing - review \& editing, Mohamed Ahmed Eladl, Mohamed ElSherbiny and Sawsan Zaitone.

Funding: This work is funded by AlMaarefa University, Saudi Arabia.

Declaration of interests: authors have no competing interests to declare.

Institutional Review Board Statement: All experiments approved by the committee for animal care and ethics at the medical college of Suez Canal University.

Informed Consent Statement: "Not applicable."

Data Availability Statement: The data of this manuscript is available upon request from FA and DI.

Acknowledgments: The authors extend their appreciation to AlMaarefa University for supporting this research. 


\section{References}

A Kato T, Yamauchi Y, Horikawa H, Monji A, Mizoguchi Y, Seki Y, Hayakawa K, Utsumi H, Kanba S (2013): Neurotransmitters, psychotropic drugs and microglia: clinical implications for psychiatry. Current medicinal chemistry 20, 331-344

Abulfadl Y, El-Maraghy N, Ahmed AE, Nofal S, Abdel-Mottaleb Y, Badary OAJH, toxicology e (2018): Thymoquinone alleviates the experimentally induced Alzheimer's disease inflammation by modulation of TLRs signaling. 37, 1092-1104

Ahmad A, Husain A, Mujeeb M, Khan SA, Najmi AK, Siddique NA, Damanhouri ZA, Anwar FJAPjotb (2013): A review on therapeutic potential of Nigella sativa: A miracle herb. 3, 337-352

Al-Ali A, Alkhawajah AA, Randhawa MA, Shaikh NAJJAMCA (2008): Oral and intraperitoneal LD50 of thymoquinone, an active principle of Nigella sativa, in mice and rats. 20, 25-27

Al-Otaibi SS, Arafah MM, Sharma B, Alhomida AS, Siddiqi NJ (2018): Synergistic effect of quercetin and $\alpha$-lipoic acid on aluminium chloride induced neurotoxicity in rats. Journal of toxicology 2018

Allison AC, Cacabelos R, Lombardi V, Alvarez X, Vigo CJCDR (2000): Central nervous system effects of celastrol, a potent antioxidant and antiinflammatory agent. 6, 45-62

Bannon D 2015: The behavioural effects of stress and aluminium toxicity on a mouse model of amyotrophic lateral sclerosis parkinsonism-dementia complex, University of British Columbia

Baydar T, Papp A, Aydin A, Nagymajtenyi L, Schulz H, Isimer A, Sahin G (2003): Accumulation of aluminum in rat brain. Biological trace element research 92, 231-244

Belaïd-Nouira Y, Bakhta H, Bouaziz M, Flehi-Slim I, Haouas Z, Cheikh HBJLih, disease (2012): Study of lipid profile and parieto-temporal lipid peroxidation in $\mathrm{AlCl} 3$ mediated neurotoxicity. Modulatory effect of fenugreek seeds. 11, 1-8

Bhalla P, Singla N, Dhawan DJB (2010): Potential of lithium to reduce aluminium-induced cytotoxic effects in rat brain. 23, 197-206

Bian M, Du X, Cui J, Wang P, Wang W, Zhu W, Zhang T, Chen YJJon (2016): Celastrol protects mouse retinas from bright light-induced degeneration through inhibition of oxidative stress and inflammation. 13, 1-15

Bove M, Tucci P, Dimonte S, Trabace L, Schiavone S, Morgese MGJFiN (2020): Postnatal Antioxidant and Anti-inflammatory Treatments Prevent Early Ketamine-Induced Cortical Dysfunctions in Adult Mice. 14, 1108

Bowdler NC, Beasley DS, Fritze EC, Goulette AM, Hatton JD, Hession J, Ostman DL, Rugg DJ, Schmittdiel CJJPB, Behavior (1979): Behavioral effects of aluminum ingestion on animal and human subjects. $10,505-512$

Brough D, Jouhara HJIJoT (2020): The aluminium industry: A review on state-of-the-art technologies, environmental impacts and possibilities for waste heat recovery. 1, 100007

Chen S, Gu C, Xu C, Zhang J, Xu Y, Ren Q, Guo M, Huang S, Chen LJJon (2014): Celastrol prevents cadmium- induced neuronal cell death via targeting JNK and PTEN- Akt/mTOR network. 128, 256-266

Dai W, Wang X, Teng H, Li C, Wang B, Wang JJIi (2019): Celastrol inhibits microglial pyroptosis and attenuates inflammatory reaction in acute spinal cord injury rats. 66, 215-223

Elmaci I, Altinoz MAJB, Pharmacotherapy (2016): Thymoquinone: An edible redox-active quinone for the pharmacotherapy of neurodegenerative conditions and glial brain tumors. A short review. 83, 635-640 
Ghorbel I, Amara IB, Ktari N, Elwej A, Boudawara O, Boudawara T, Zeghal NJBter (2016): Aluminium and acrylamide disrupt cerebellum redox states, cholinergic function and membrane-bound ATPase in adult rats and their offspring. 174, 335-346

Gitler AD, Dhillon P, Shorter J (2017): Neurodegenerative disease: models, mechanisms, and a new hope. The Company of Biologists Ltd

Golub MS, Germann SLJN, teratology (2001): Long-term consequences of developmental exposure to aluminum in a suboptimal diet for growth and behavior of Swiss Webster mice. $23,365-372$

Han S, Lemire J, Appanna VP, Auger C, Castonguay Z, Appanna VDJCB, Toxicology (2013): How aluminum, an intracellular ROS generator promotes hepatic and neurological diseases: the metabolic tale. $29,75-84$

Hori K, Konishi K, Akita R, Tani M, Tomioka H, Kitajima Y, Yokoyama S, Azuma K, Ikuse D, Akashi NJNssyzJjop (2013): Proposal of endogenous anticholinergic hypothesis in Alzheimer disease. 33, 117-126

Hosseinzadeh H, Taiari S, Nassiri-Asl MJN-Ssaop (2012): Effect of thymoquinone, a constituent of Nigella sativa L., on ischemia-reperfusion in rat skeletal muscle. 385, 503-508

Ince S, Kucukkurt I, Demirel HH, Turkmen R, Zemheri F, Akbel EJT, Chemistry E (2013): The role of thymoquinone as antioxidant protection on oxidative stress induced by imidacloprid in male and female Swiss albino mice. 95, 318-329

Justin-Thenmozhi A, Bharathi MD, Kiruthika R, Manivasagam T, Borah A, Essa MMJNr (2018): Attenuation of aluminum chloride-induced neuroinflammation and caspase activation through the AKT/GSK-3 $\beta$ pathway by hesperidin in wistar rats. 34, 463-476

Kassab RB, El-Hennamy REJEJoB, Sciences A (2017): The role of thymoquinone as a potent antioxidant in ameliorating the neurotoxic effect of sodium arsenate in female rat. 4, 160167

Khan KA, Kumar N, Nayak PG, Nampoothiri M, Shenoy RR, Krishnadas N, Rao CM, Mudgal J (2013): Impact of caffeic acid on aluminium chloride-induced dementia in rats. The Journal of pharmacy and pharmacology 65, 1745-52

Kumar SJF, toxicology c (2002): Aluminium-induced changes in the rat brain serotonin system. $40,1875-1880$

Li J, Hao JJTAjoCm (2019): Treatment of neurodegenerative diseases with bioactive components of Tripterygium wilfordii. 47, 769-785

Majdalawieh AF, Hmaidan R, Carr RI (2010): Nigella sativa modulates splenocyte proliferation, Th1/Th2 cytokine profile, macrophage function and NK anti-tumor activity. Journal of ethnopharmacology 131, 268-275

Metz GA, Tse A, Ballermann M, Smith LK, Fouad K (2005): The unilateral 6- OHDA rat model of Parkinson's disease revisited: an electromyographic and behavioural analysis. European Journal of Neuroscience 22, 735-744

Milnerowicz H, Ściskalska M, Dul MJJoTEiM, Biology (2015): Pro-inflammatory effects of metals in persons and animals exposed to tobacco smoke. 29, 1-10

Nakayama T, Okimura K, Shen J, Guh Y-J, Tamai TK, Shimada A, Minou S, Okushi Y, Shimmura T, Furukawa YJPotNAoS (2020): Seasonal changes in NRF2 antioxidant pathway regulates winter depression-like behavior. 117, 9594-9603

Paris D, Ganey NJ, Laporte V, Patel NS, Beaulieu-Abdelahad D, Bachmeier C, March A, AitGhezala G, Mullan MJJJon (2010): Reduction of $\beta$-amyloid pathology by celastrol in a transgenic mouse model of Alzheimer's disease. 7, 1-15 
Peng J-HF, Xu Z-C, Xu Z-X, Parker JC, Friedlander ER, Tang J-P, Melethil SJM, neuropathology c (1992): Aluminum-induced acute cholinergic neurotoxicity in rat. 17, 79-89

Rashwan EH, Kamel MM, El-lethey HS, Ciobica A, El Iraqi KG, Ahmed-Farid OAJIJoPR, Sciences A (2018): Caffeine Ameliorating Effect on Anxiety and Depression in an Aluminum Chloride-induced Alzheimer's Disease Rat Model. 7

Ravi S, Prabhu B, Raju T, Bindu PJIjop, pharmacology (2000): Long-term effects of postnatal aluminium exposure on acetylcholinesterase activity and biogenic amine neurotransmitters in rat brain. 44, 473-478

Safhi MMJApp (2016): NEUROMODULATORY EFFECTS OF THYMOQUINONE IN EXTENUATING OXIDATIVE STRESS IN CHLORPROMAZINE TREATED RATS. $73,529-535$

Skalny A, Aschner M, Jiang Y, Gluhcheva Y, Tizabi Y, Lobinski R, Tinkov A (2021): Molecular mechanisms of aluminum neurotoxicity: Update on adverse effects and therapeutic strategies

Spanos M, Gras-Najjar J, Letchworth JM, Sanford AL, Toups JV, Sombers LAJAcn (2013): Quantitation of hydrogen peroxide fluctuations and their modulation of dopamine dynamics in the rat dorsal striatum using fast-scan cyclic voltammetry. 4, 782-789

Troshin VJMtipe (2009): Pathogenesis and classification of chronic encephalopathy due neurotoxic chemicals (review of literature). 21-26

Yadid G, Pacak K, Kopin IJ, Goldstein DSJJoP, Therapeutics E (1994): Endogenous serotonin stimulates striatal dopamine release in conscious rats. 270, 1158-1165

Yokel RAJN (2000): The toxicology of aluminum in the brain: a review. 21, 813-828

Zghari O, Rezqaoui A, Ouakki S, Lamtai M, Chaibat J, Mesfioui A, El Hessni A, Rifi E-H, Essamri A, Ouichou AJJoB, science b (2018): Effect of chronic aluminum administration on affective and cognitive behavior in male and female rats. 8, 179-196

Zhang R, Zhu Y, Dong X, Liu B, Zhang N, Wang X, Liu L, Xu C, Huang S, Chen LJJocp (2017): Celastrol Attenuates Cadmium- Induced Neuronal Apoptosis via Inhibiting Ca2+CaMKII- Dependent Akt/mTOR Pathway. 232, 2145-2157

Zhu C, Yang J, Zhu Y, Li J, Chi H, Tian C, Meng Y, Liu Y, Wang J, Lin NJC, medicine t (2021): Celastrol alleviates comorbid obesity and depression by directly binding amygdala HnRNPA1 in a mouse model. 11 


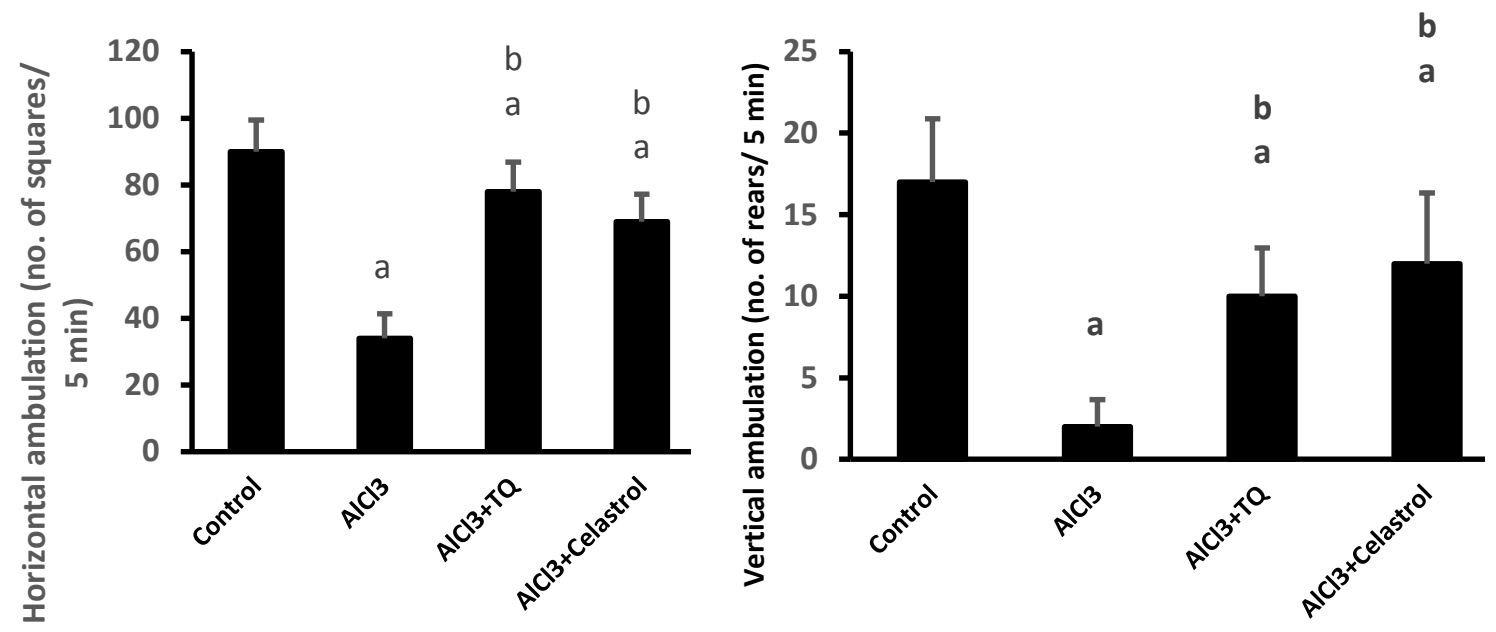

Fig 1. Motor activity of rats in the open field test. A) Horizontal ambulation and B) vertical ambulation. Results were expressed as mean \pm SD and analyzed using one-way ANOVA followed by Bonferroni's post-hoc test at $P<0.05 .^{\text {a, b }}$ Represents a statistically significant difference when compared to control and $\mathrm{AlCl} 3$, respectively. 


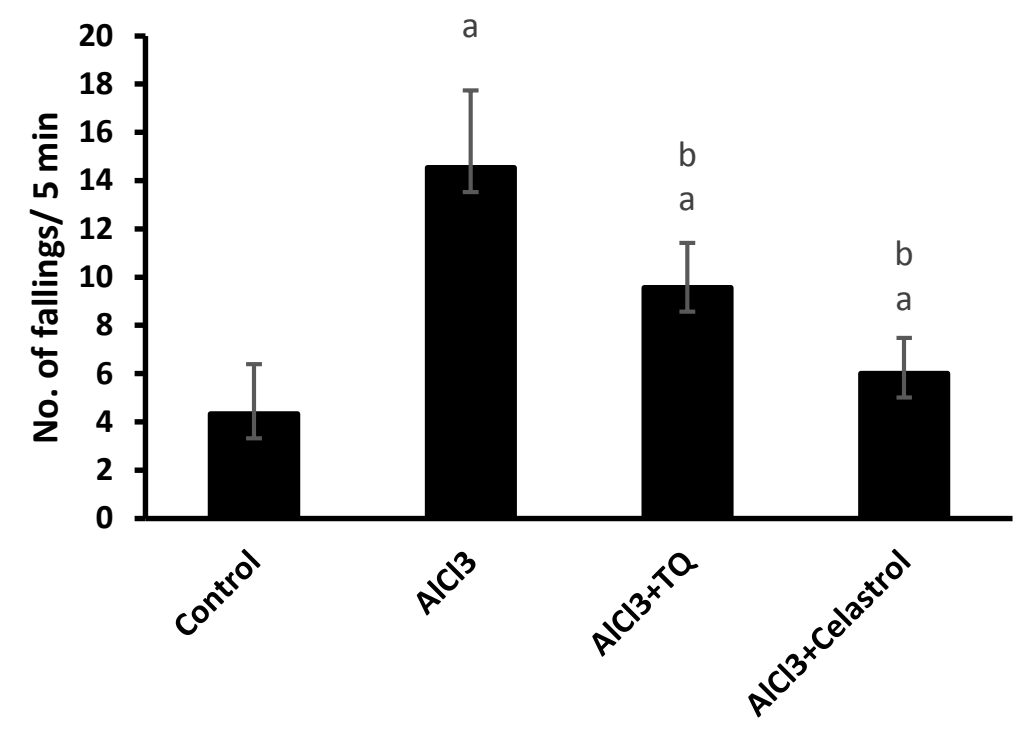

Fig 2. Number of fallings in the rotarod test. Results were expressed as mean \pm SD and analyzed using oneway ANOVA followed by Bonferroni's post-hoc test at $\mathrm{P}<0.05$. a, $\mathrm{b}$ and $\mathrm{c}$ Represents a statistically significant difference when compared to control, $\mathrm{AlCl} 3$ and $\mathrm{AlCl} 3+\mathrm{TQ}$, respectively. 


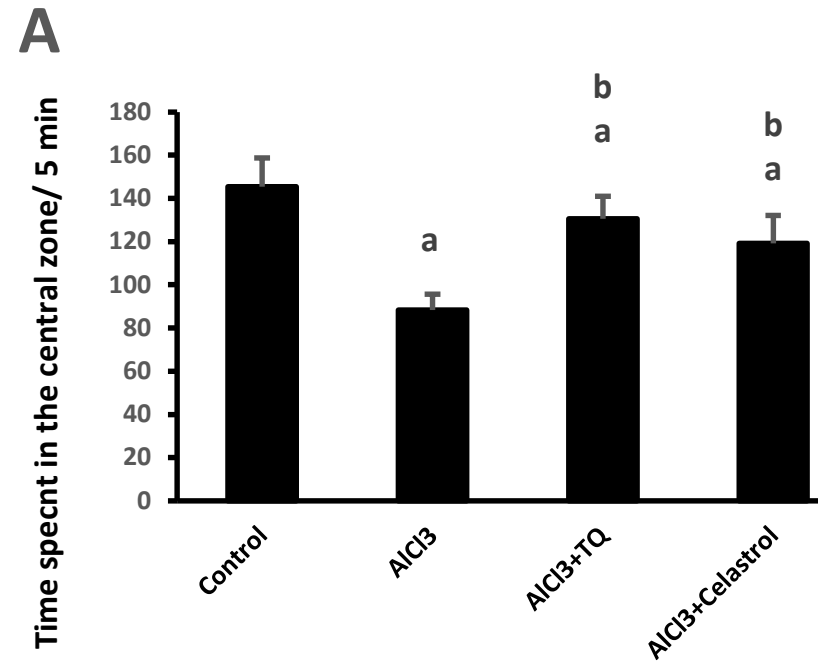

B

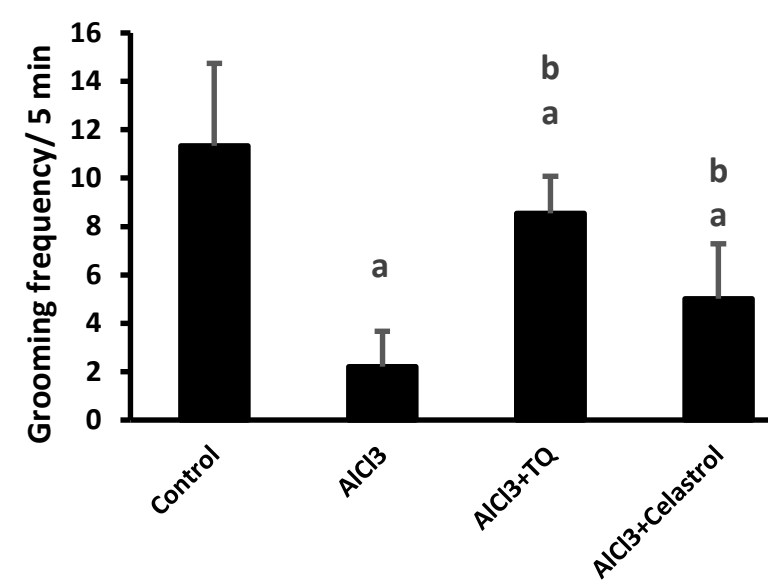

Fig 3. Anxiety-like behavioral parameters in the open field test. A) Time spent in the central zone \& B) Grooming frequency/ 5 min. Results were expressed as mean \pm SD and analyzed using one-way ANOVA followed by Bonferroni's post-hoc test at $\mathrm{P}<0.05$. a, b and c Represents a statistically significant difference when compared to control, $\mathrm{AlCl} 3$ and $\mathrm{AlCl} 3+\mathrm{TQ}$, respectively. 


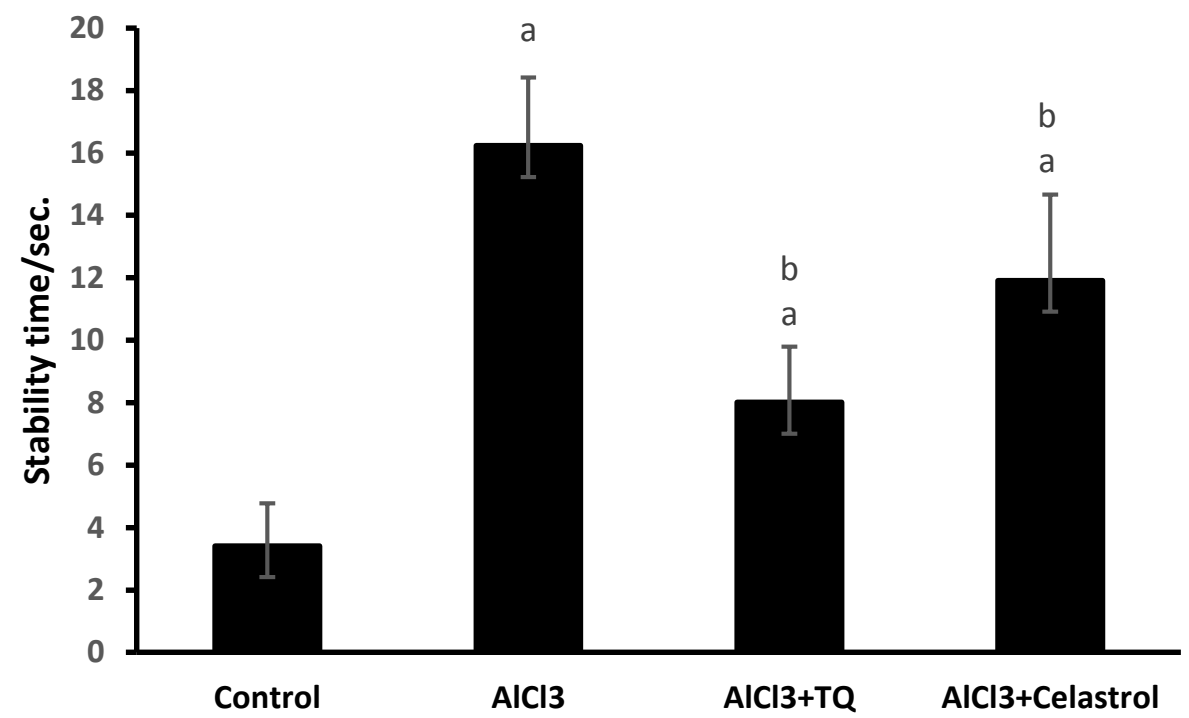

Fig 4. Depressive-like behaviors in the rat forced swimming test. Results were expressed as mean \pm SD and analyzed using one-way ANOVA followed by Bonferroni's post-hoc test at $\mathrm{P}<0.05$. a, b represents a statistically significant difference when compared to control and $\mathrm{AlCl} 3$, respectively 